\section{Características da Aids na terceira idade em um hospital de referência do Estado do Ceará, Brasil}

\section{Characteristics of Aids amongst the elderly at a reference hospital in the State of Ceará, Brazil}

Vera Lúcia Borges de Araújo

Daniele Mary Silva de Brito ${ }^{2}$

Marli Teresinha Gimeniz ${ }^{2}$

Terezinha Almeida Queiroz ${ }^{3}$

Clodis Maria Tavares ${ }^{4}$

${ }^{1}$ Hospital São José em Doenças Infecciosas

${ }^{2}$ Faculdade de Enfermagem da Universidade Federal do Ceará

${ }^{3}$ Faculdade de Enfermagem da Universidade Estadual do Ceará

${ }^{4}$ Universidade de Fortaleza

Correspondência: Vera Lúcia Borges de Araújo. Av. Jovita Feitosa 2326 Apto. 13 - Parquelândia, Fortaleza, CE - CEP: 60455-410. E-mail: veraborges-ar@oi.com.br

\section{Resumo}

Idosos contaminados pelo HIV ampliam as estatísticas ao redor do mundo. Este estudo teve como objetivo geral descrever as características da Aids em pessoas com 60 anos de idade ou mais, atendidos em hospital de referência para HIV/Aids no Ceará, no período de 1989 a 2004. Desenvolveu-se pesquisa documental utilizando fichas de notificação compulsória de Aids disponíveis no Sistema de Informação de Agravos de Notificação (SINAN) e dados do Sistema de Informação sobre Mortalidade (SIM). Obteve-se notificação de 107 casos, dos quais 50 encontravam-se vivos e 57 foram a óbito. Pela análise observou-se crescimento progressivo dos casos, que, em sua maioria $(77,5 \%)$, concentravam-se em Fortaleza. A faixa etária mais comprometida foi a de 60 a 69 anos (77,5\%). Grande parte dos indivíduos mostrou baixo nível de escolaridade (44,0\%). A subcategoria heterossexual aumentou ao longo do tempo. Evidenciou-se maior letalidade entre as mulheres $(73,9 \%)$. O conjunto de dados analisados permite inferir que entre a população acima de 60 anos com Aids no Ceará verifica-se tendência semelhante às demais faixas etárias e fenômenos observados no país, a saber: heterossexualização, feminização, envelhecimento, pauperização.

Palavras-chave: HIV-1. Síndrome de imunodeficiência adquirida. Idosos. Terceira idade. Epidemiologia. 


\section{Abstract}

Elderly people contaminated by the HIV are increasing statistics throughout the world. This study's main aim was to describe the characteristics of AIDS in people aged 60 and over, seen at a benchmark hospital for HIV / AIDS in Ceará, Brazil, in the period between 1989 and 2004. Documentary research was performed using compulsory AIDS reporting forms available on the National Reporting Information System (SINAN) and data from the Mortality Information System (SIM). There were 107 cases reported, of which 50 patients were still alive and 57 deceased. Based on the analysis, a progressive growth in cases was observed, most of which (77.5\%) were concentrated in Fortaleza. The most affected age group was the one between 60 and 69 (77.5\%). Many of the individuals showed low level of schooling (44.0\%). The subcategory of heterosexuals grew over time. Mortality amongst women was found to be higher (73.9\%). The set of data analyzed enables one to infer that the population over 60 with AIDS in Ceará shows similar trends to other age groups and the phenomena observed throughout the country, that is: growth in cases among heterosexuals and women, and ageing and impoverishment.

Keywords: HIV -1. Acquired Immune Deficiency Syndrome. Elderly. Senior Citizens. Epidemiology.

\section{Introdução}

A Aids compromete indivíduos de todas as faixas etárias. No Brasil, dos 371.827 casos notificados de 1980 até junho de 2005, o número de pessoas entre 50 e 59 anos representou $6,2 \%$ da totalidade dos casos e em indivíduos com 60 anos ou mais: $2,1 \%$ do total entre os sexos ${ }^{1}$.

Em nosso país, especialmente nos últimos anos, observa-se que a porcentagem dos pacientes com 50 anos ou mais no diagnóstico de Aids aumentou progressivamente de 7\% em 1996 para 13\% em 2004².

$\mathrm{O}$ crescimento do número de infecções por HIV/Aids em pessoas com 60 anos ou mais resulta na mais nova característica da epidemia. Faz-se necessário campanhas de prevenção à DST/Aids direcionadas a esse segmento, proporcionando assim qualidade de vida para os idosos ${ }^{3}$.

No Estado do Ceará, o primeiro caso de Aids registrado ocorreu em 1983, paciente oriundo do sudeste do País. Dados acumulados até 2004 indicam 5.763 casos notificados. A Secretaria da Saúde do Estado do Ceará (SESA) também contabilizou, do início da epidemia de Aids na terceira idade até 2004, 116 casos da doença.

Sabe-se que o primeiro caso de Aids do Ceará na terceira idade é datado de 1989, paciente do sexo masculino e forma de infecção sexual ${ }^{4}$.

Atribuem-se dois fatores como responsáveis pelo aumento de casos de Aids em idades mais avançadas. $\mathrm{O}$ primeiro devese àqueles idosos que possuem, entre outros fatores, maiores recursos, o que contribui para o acesso aos prazeres e serviços disponíveis, permitindo vida sexual mais ativa. O segundo fator deve-se, principalmente, à existência de tabu sobre sexualidade na terceira idade ${ }^{5}$.

É enganoso, porém, pensar que as pessoas idosas não fazem sexo e não usam drogas, a despeito de poucas campanhas de prevenção dirigidas a essa população. Portanto, de modo geral, estas pessoas estão menos informadas sobre o HIV e pouco conscientes de como se protegerem. 
Desse modo, tomam-se vulneráveis à infecção ${ }^{4}$. Velhice sem sexualidade é um mito ${ }^{7}$.

No Brasil, os casos de infecção de Aids na faixa etária de mais de 60 anos acontecem predominantemente por transmissão sexual. Em virtude da estigmatização da terceira idade, tanto os familiares como os profissionais negam-se a pensar que nesta fase a pessoa está ativa sexualmente. Essa falha traz graves conseqüências, sobretudo quanto à prevenção, pois esta só vai ocorrer se os familiares e profissionais de saúde estiverem atentos para discutir abertamente as formas de prevenção.

É difícil determinar os índices de infecção pelo HIV. Até os profissionais ainda são reticentes e raramente indagam sobre a vida sexual do idoso, nem suspeitam da possibilidade da contaminação pelo HIV e retardam o diagnóstico. Embora muitas vezes sintomas como desidratação, fraqueza, anorexia e febre sejam causas freqüentes de internação em idosos, podem levar os médicos a fazerem um diagnóstico incompleto.

Em 1995, em um hospital de Nova York, ao avaliar um grupo de 257 idosos infectados pelo HIV no período de um ano, pesquisadores já constatavam subnotificação de 5,05\% (13 pacientes) em relação aos óbitos por HIV. A morte de nenhum destes foi atribuída 'a infecção pelo $\mathrm{HIV}^{8}$.

Como mostram os dados, a epidemia de HIV/Aids configura nas pessoas desta faixa etária um dos mais sérios problemas contemporâneos de saúde pública e apresenta alto grau de morbimortalidade, perspectivas de contínuo crescimento e propagação em todos os continentes. Se não forem tomadas medidas sérias de prevenção e contenção da doença, as possibilidades de controle da epidemia serão remotas, não obstante o desenvolvimento de novas terapias e os esforços mundiais na busca de uma vacina eficaz contra a infecção ${ }^{9}$.

Diante da perspectiva do aumento do número de idosos infectados na terceira idade, e em decorrência da ampliação da expectativa de vida facilitada pelos antiretrovirais e pela descoberta de novos casos nesta faixa etária, faz-se necessário se conhecer características regionais dessa população já comprometida.

Em face do exposto, elaborou-se este estudo, cujo objetivo geral é descrever as características dos pacientes de Aids de terceira idade atendidos no hospital de referência para HIV/Aids no Estado do Ceará.

\section{Métodos}

No presente estudo, será utilizada para idoso a definição da Organização Mundial de Saúde (OMS), que especifica como população idosa aquela a partir de $60 \operatorname{anos}^{10}$.

Desenvolveu-se estudo descritivo retrospectivo para caracterizar a epidemia de Aids no hospital de referência do Ceará. Como fonte de dados foram utilizadas fichas de notificação compulsória armazenadas no núcleo de vigilância epidemiológica do Hospital São José de Doenças Infecciosas as quais são processadas pelo Sistema de Informação de Agravos de Notificação (SINAN). Estas informações foram validadas pela Vigilância Epidemiológica da Secretaria de Estado da Saúde do Ceará.

Foram considerados e estudados os casos de Aids notificados na instituição, por ano de diagnóstico, entre $1^{\circ}$ de janeiro de 1989 e 31 de dezembro de 2004. A população de estudo constituiu-se de 107 casos de indivíduos com 60 anos ou mais de idade, atendidos e notificados no serviço. Todos os casos foram estudados. Este número correspondeu a $92,2 \%$, num total de 116 casos nesta faixa etária no Estado.

As variáveis abordadas neste estudo foram as seguintes: sexo, idade, escolaridade (anos de estudo), número de habitantes do município de procedência, categoria de exposição e ano do diagnóstico. Os dados populacionais foram reunidos de censos demográficos e de projeções e estimativas por grupo de idade, acessadas por meio eletrônico, como no site www.datasus.gov.br/cgi/tabecgi.exe?ibge/ cnv/pop.ce.def, em 20/09/2005. Também 
foi observada a ocorrência de mortes nessa população. Para isto utilizou-se o Sistema de Informação de Mortalidade (SIM), acessando o site www.datasus.gov.br, em 28/06/2005, observando-se os óbitos por Aids como causa mortis nessa população. O código 279.1 da 9a revisão de Classificação Internacional de Doenças(CID) foi utilizado para os anos de 1989 a 1995 e os códigos B20 e B24 da 10ª revisão da CID para os anos de 1996 a 2004 ${ }^{11-14}$.

Foi estabelecida ligação entre os bancos de dados do SINAN e do SIM.

Os dados foram apresentados em forma de tabelas e gráficos.

O processo adotado na análise dos dados envolveu medidas de razão de sexos, coeficientes de incidência e letalidade.

Os coeficientes de incidência representam, por definição, medidas por excelência do risco de doença e de agravo, e o de letalidade permite avaliar a gravidade de uma doença.

Os valores calculados de incidência e letalidade obedeceram as fórmulas recomendadas para estudos epidemiológicos ${ }^{15}$.

Este estudo teve aprovação do Comitê de Ética em Pesquisa do Hospital lócus da pesquisa, conforme recomendado pela Resolução no 196/96 do Conselho Nacional de Saúde ${ }^{16}$.

\section{Resultados}

No período de 1989 a 2004, no hospital em estudo, o número de casos de Aids em indivíduos com 60 anos ou mais incidiu em 107 pessoas, das quais $84(78,5 \%)$ eram homens e 23 (21,5\%) mulheres.

Nesta pesquisa, entre os idosos notificados como portadores de Aids, o grupo etário mais acometido foi aquele com idades entre 60 e 69 anos (77,5\%). Além disso, $6,7 \%$ dos casos comprometiam indivíduos com 80 anos ou mais (Tabela 1).

Quanto à escolaridade dos idosos, observou-se desde analfabetos até aqueles que cursaram o ensino superior. Conforme se percebe, as mulheres apresentaram menor ingresso no ambiente escolar, sen- do $21,7 \%$ delas sem escolaridade. Entre os sexos destaca-se elevado percentual de ignorados $(36,4 \%)$. (Tabela 1$)$

A Tabela 1 também demonstra os casos de Aids entre idosos por número de habitantes, os quais estiveram distribuídos entre municípios de pequeno porte $(<$ 50.000 habitantes) até a capital do Estado, com população acima de 1.000 .000 habitantes concentrando $77,5 \%$ da epidemia.

Em relação à categoria de exposição, a maioria $(42,9 \%)$ dos homens foi notificada na subcategoria homo-bissexual, seguida pela forma heterossexual (34,5\%). Entre as mulheres, a maior porcentagem $(56,5 \%)$ ocorreu por transmissão heterossexual. Houve apenas uma contaminação por UDI (usuário de drogas injetáveis) entre os homens. Entre os sexos, observa-se alto percentual de notificação classificada como "ignorada” (Tabela 1).

Os casos de Aids entre os idosos, ano a ano, por categoria de exposição no presente estudo, foram apresentados por ano de ocorrência como mostra o Gráfico 1. Quanto à forma de contaminação pelo HIV, no sexo masculino, de 1989 a 1993, todos os casos diagnosticados pertenciam à subcategoria homo-bissexual (100,0\%). A partir de 1998, no entanto, verifica-se o aumento e permanência dos casos entre os sexos na subcategoria heterossexual.

Neste estudo, os idosos apresentaram $53,2 \%$ de mortes em decorrência da Aids (Tabela 1).

\section{Incidência}

Para se compreender melhor a importância deste estudo no Ceará, calculamos a incidência de Aids na terceira idade mostrando a série histórica do Ceará e do Hospital de referência para uma melhor visualização da epidemia.

No Gráfico 2 observa-se que, de um modo geral, a incidência de casos de Aids na terceira idade no Estado do Ceará e no Hospital de Referência apresenta valores praticamente iguais na maioria dos anos.

Entre os homens houve um aumento 
Tabela 1 - Casos de Aids em indivíduos com 60 anos de idade ou mais, segundo variáveis sóociodemográfico, categoria de exposição e de óbitos $(n=107)$. Hospital de Referência Ceará. 1989 - 2004.

Table 1 - AIDS cases in individuals over 60, according to sociodemographic variables, exposure category and deaths ( $n=107)$. Reference Hospital in Ceará 1989-2004

\begin{tabular}{|c|c|c|c|c|c|c|}
\hline \multirow[t]{2}{*}{ Variáveis } & \multicolumn{2}{|c|}{ Masculino $(n=84)$} & \multicolumn{2}{|c|}{ Feminino $(n=23)$} & \multicolumn{2}{|c|}{ Total } \\
\hline & $\mathrm{N}^{\circ}$ & $\%$ & $\mathrm{~N}^{\circ}$ & $\%$ & $\mathrm{~N}^{\circ}$ & $\%$ \\
\hline \multicolumn{7}{|l|}{ Faixa etária (anos) } \\
\hline $60-69$ & 67 & 80,0 & 16 & 70,0 & 83 & 77,5 \\
\hline $70-79$ & 14 & 16,6 & 3 & 12,0 & 17 & 15,8 \\
\hline 80 & 3 & 3,4 & 4 & 7,0 & 7 & 6,7 \\
\hline \multicolumn{7}{|l|}{ Anos de estudo } \\
\hline Nenhum & 10 & 11,9 & 5 & 21,7 & 15 & 14,0 \\
\hline Fundamental (8anos) & 24 & 28,6 & 8 & 34,8 . & 32 & 30,0 \\
\hline Médio/superior(8anos) & 20 & 23,8 & 1 & 4,4 & 21 & 19,6 \\
\hline Ignorado & 30 & 35,7 & 9 & 39,1 & 39 & 36,4 \\
\hline \multicolumn{7}{|l|}{$\begin{array}{l}\mathrm{N}^{\circ} \text { de habitantes } \\
\text { (Município de procedência) }\end{array}$} \\
\hline$<50.000$ & 8 & 7.4 & 2 & 1.8 & 10 & 9.3 \\
\hline $50.000-200.000$ & 9 & 8.4 & 3 & 2.8 & 12 & 11.2 \\
\hline $200.001-500.000$ & 1 & 0.9 & 1 & 0.9 & 2 & 1.8 \\
\hline \multicolumn{7}{|l|}{$500.001-1.000 .000$} \\
\hline * .1 .000 .001 & 66 & 61.6 & 17 & 15.8 & 83 & 77.5 \\
\hline \multicolumn{7}{|l|}{ Categoria de exposição } \\
\hline Homo/Bissexual & 36 & 42,9 & - & - & 36 & 33,7 \\
\hline Heterossexual & 29 & 34,5 & 13 & 56,5 & 42 & 34,3 \\
\hline U.D.I. & 1 & 1,2 & - & - & 1 & 0,9 \\
\hline lgnorada & 18 & 21,4 & 10 & 43,5 & 28 & 26,1 \\
\hline \multicolumn{7}{|l|}{ Óbitos } \\
\hline Sim & 40 & 47,6 & 17 & 73,9 & 57 & 53,2 \\
\hline Não & 44 & 52,4 & 6 & 26,1 & 50 & 46,8 \\
\hline
\end{tabular}

Fonte: SESA/SINAM/SIM

* Fortaleza

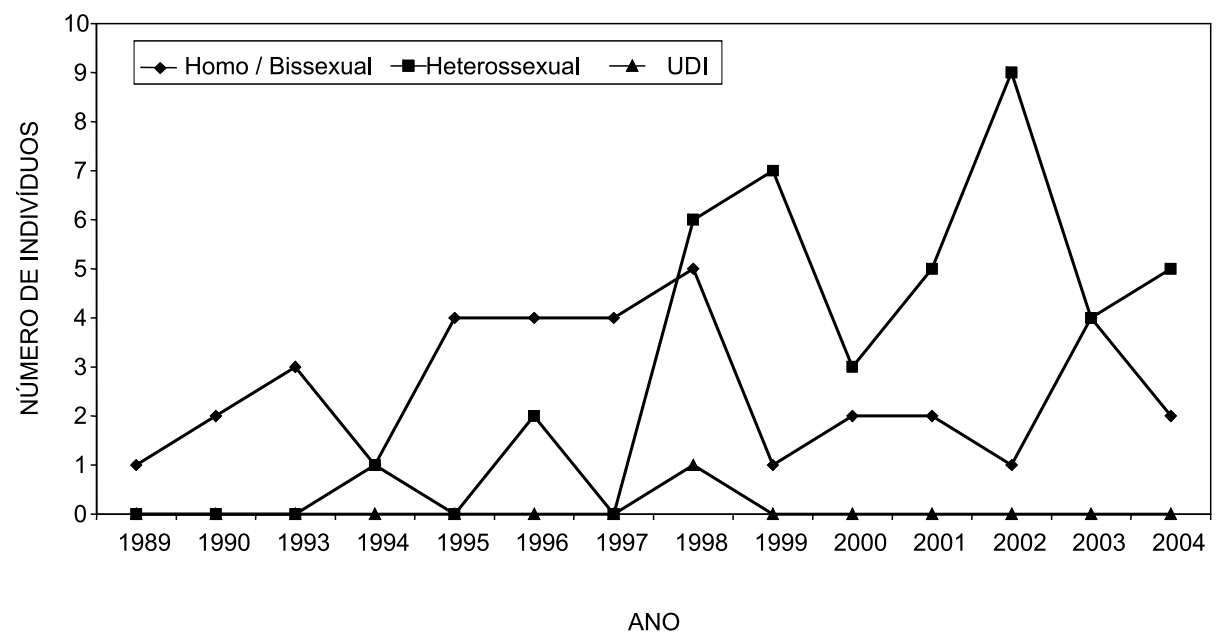

Gráfico 1 - Casos de Aids em indivíduos com 60 anos de idade ou mais, segundo categoria de exposição e ano de diagnóstico. Hospital de Referência do Ceará. 1989 - 2004.

Graph 1 - AIDS cases in individuals over 60, according to exposure category and year of diagnosis. Reference Hospital in Ceará. 1989 - 2004. 


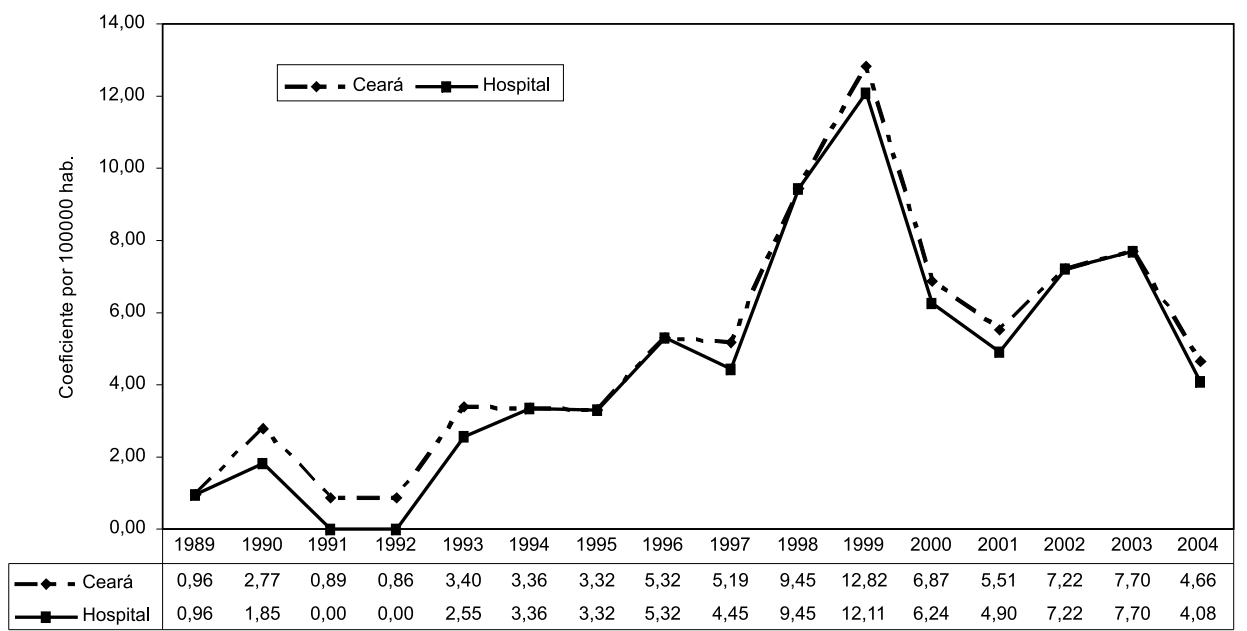

Fonte: SESA/SINAN

Gráfico 2 - Incidência de Aids em indivíduos com 60 anos de idade ou mais, por ano de diagnóstico e segundo Estado do Ceará e Hospital de Referência do Ceará por Hospital de Referência do Ceará, 1989 - 2004.

Graph 2 - Incidence of AIDS in individuals over 60, according to year of diagnosis and State of Ceará and Reference Hospital in Ceará by Reference Hospital in Ceará. 1989 - 2004.

da incidência a partir de 1993 (6,28 por 100.000 hab.). Em 1996 este se mostra de forma expressiva (10,88 por 100.000 hab.), alcançando o ápice em 1998, com uma incidência de 18,78 por 100.000 hab., mantendo-se em patamares elevados até 2003 (14,30 por 100.000hab.) Em relação às mulheres, em 1999 a epidemia atinge o pico
(9,09 por 100.000 hab., apresentando um ligeiro declínio em 2003, com uma incidência de 2,78 por 100.000 hab.

\section{Razão entre os sexos}

Pelo estudo da proporção dos casos entre os sexos (Razão: Homem/Mulher)

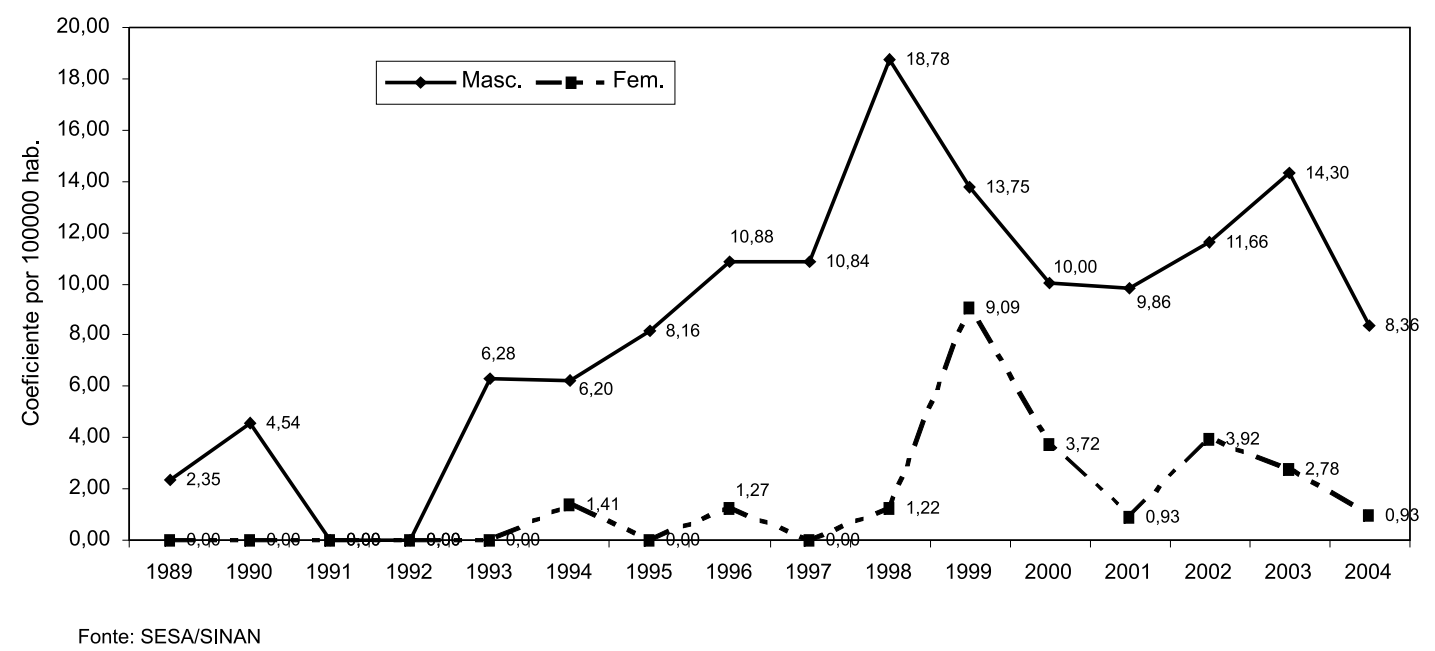

Gráfico 3 - Incidência de Aids em indivíduos com 60 anos de idade ou mais, por ano de diagnóstico e segundo sexo. Hospital de Referência do Ceará. 1989 - 2004.

Graph 3 - Incidence of AIDS in individuals over 60, according to year of diagnosis and gender. Reference Hospital in Ceará. $1989-2004$. 
houve ampla oscilação quanto à razão entre os sexos. O primeiro caso de Aids do sexo feminino em idosos, em Fortaleza, ocorreu no ano de 1994. De 1989 até 1993 verificou-se razão nula, enquanto em 1998 observou-se razão 12:1. Em 2004, mantémse elevada com 6H:1M (Tabela 2).

\section{Coeficiente de letalidade}

Em relação à letalidade de Aids na população com idade igual ou superior a 60 anos no estudo, percebe-se, durante os primeiros anos da epidemia, letalidade de $100 \%$, embora, a partir de 1996, possa ser visualizada uma queda no coeficiente de letalidade. Essa redução coincide com o início da utilização da terapia anti-retroviral no país.

A mortalidade por Aids em mulheres atingiu o máximo em 1999. Contudo, entre os anos de 2000 e 2003, mostrou certa estabilização.

\section{Discussão}

A propagação da Aids no Brasil evidencia uma epidemia de múltiplas dimensões que, ao longo do tempo, tem apresentado profundas transformações na sua evolução e distribuição. Vista a princípio como uma epidemia específica de indivíduos jovens e considerados de "grupos de risco" , passou a atingir qualquer indivíduo da sociedade, independente de sexo e idade.

No Brasil, concomitantemente à expansão da infecção pelo HIV, os portadores têm sido beneficiados pelo acesso gratuito aos anti-retrovirais, que proporcionam maior chance de sobrevivência, mesmo no caso de idades antes não alcançadas.

\section{Envelhecimento}

No início da década de 1990 (a partir de 1994 e 1995), no Brasil, a epidemia de Aids na faixa de 50 a 70 anos mostrou leve

Tabela 2 - Casos de Aids, óbitos e letalidade informada em indivíduos com 60 anos de idade ou mais, segundo ano de diagnóstico, por sexo e razão de sexo. Hospital de Referência do Ceará, 1989 - 2004.

Table 2 - AIDS cases, deaths and informed lethality in individuals over 60 years of age, by year of diagnosis, gender and sex ratio. Reference Hospital in Ceará. 1989- 2004.

\begin{tabular}{|c|c|c|c|c|c|c|c|c|c|c|c|}
\hline \multirow[t]{2}{*}{ Ano } & \multicolumn{4}{|c|}{ Casos (No) } & \multicolumn{4}{|c|}{ Óbitos (no) } & \multicolumn{3}{|c|}{ Letalidade informada (\%) } \\
\hline & Masc & Fem & Total & MF & Masc & Fem & Total & MF & Masc & Fem & Total \\
\hline 1989 & 1 & - & 1 & - & 1 & - & 1 & - & 100,0 & - & 100,0 \\
\hline 1990 & 2 & - & 2 & - & - & - & - & - & - & - & - \\
\hline 1991 & - & - & - & - & - & - & - & - & - & - & - \\
\hline 1992 & - & - & - & - & 1 & - & 1 & - & 100,0 & - & 100,0 \\
\hline 1993 & 3 & - & 3 & - & 3 & - & 3 & - & 100,0 & - & 100,0 \\
\hline 1994 & 3 & 1 & 4 & 3,0 & 1 & - & 1 & - & 33,3 & - & 25,0 \\
\hline 1995 & 4 & - & 4 & - & 4 & - & 4 & - & 100,0 & - & 100,0 \\
\hline 1996 & 6 & 1 & 7 & 6,0 & 1 & - & 1 & - & 16,6 & - & 14,2 \\
\hline 1997 & 6 & - & 6 & - & 2 & - & 2 & - & 33,3 & - & 33,3 \\
\hline 1998 & 12 & 1 & 13 & 12,0 & 5 & 2 & 7 & 2,5 & 41,6 & 16,6 & 53,8 \\
\hline 1999 & 9 & 8 & 17 & 1,1 & 3 & 7 & 10 & 0,4 & 33,3 & 87,5 & 58,8 \\
\hline 2000 & 7 & 3 & 10 & 2,3 & 5 & 3 & 8 & 1,6 & 71,4 & 100,0 & 80,0 \\
\hline 2001 & 7 & 1 & 8 & 7,0 & 2 & - & 2 & - & 28,5 & - & 25,0 \\
\hline 2002 & 8 & 4 & 12 & 2,0 & 4 & 2 & 6 & 2,0 & 50,0 & 50,0 & 50,0 \\
\hline 2003 & 10 & 3 & 13 & 3,3 & 6 & 3 & 9 & 2,0 & 60,0 & 100,0 & 69,2 \\
\hline 2004 & 6 & 1 & 7 & 6,0 & 2 & - & 2 & - & 33,3 & - & 28,5 \\
\hline Total & 84 & 23 & 107 & 3,6 & 40 & 17 & 57 & 2,3 & 47,6 & 73,9 & 53,2 \\
\hline
\end{tabular}

Fonte: SESA/SINAN/SIM 
aumento, ao contrário do ocorrido em outras faixas etárias nas quais houve estabilização. Diante desta situação, e no intuito de. compreender o comprometimento de pessoas idosas, pesquisadores indagam, com diversos questionamentos, conforme exposto a seguir: Poderiam essas mudanças terem sido provocadas por diversos fatores ligados à sexualidade, como questões culturais, baixa noção de riscos, prática de sexo sem proteção, heterossexualização e feminização, terapias mais eficazes, aumento de expectativa de vida e aumento da atividade sexual? É possível que, com maior expectativa de vida e vida mais ativa, a sexualidade seja promovida entre os idosos, e resulte em ampliação das relações sexuais, provavelmente sem uso de preservativo $^{17,18}$ ?

A mencionada situação do aumento de indivíduos com idades mais velhas do que se via normalmente também era alvo de atenção de outros profissionais. Segundo informações, pesquisadores chamavam a atenção para o aumento da incidência de Aids na faixa etária de 60 a 69 anos, no Brasil, a partir de $1995^{18}$.

A incidência da Aids no Brasil, na faixa etária de 60 a 69 anos, passou de 6,84 casos por 100.000 habitantes, em 1990, para 18,74 casos por 100.000 habitantes em 1998. A partir deste período houve crescimento neste grupo de idade. Essa mesma tendência foi evidenciada no presente estudo. Entretanto, conforme referido, campanhas educativas e de prevenção específica para fases mais avançadas da vida não têm sido promovidas no país.

\section{Feminização e heterossexualização}

No Brasil a via de transmissão heterossexual constitui a mais importante característica da dinâmica da epidemia. Este atributo tem contribuído decisivamente para o aumento de casos em mulheres. Segundo ressaltado, a inserção da mulher na epidemia de Aids não se restringe à faixa etária mais jovem, pois tem sido observado aumento das notificações em idades mais avançadas, a partir dos 50 anos. A tendência de crescimento do número de mulheres na epidemia, sobretudo na subcategoria heterossexual, desde 1992, traduzse na maior vulnerabilidade feminina e menor acesso aos serviços de saúde reprodutiva, além da dificuldade em negociar o uso de preservativo com o parceiro na maioria dos $\operatorname{casos}^{19}$.

As mudanças do processo natural de envelhecimento entre as mulheres, como estreitamento vaginal, diminuição da elasticidade e das secreções vaginais e o desgaste das paredes vaginais, são situações que favorecerem o risco da infecção pelo HIV durante as relações sexuais. Essa situação, associada à ausência da percepção de risco, pode conduzir um número maior de mulheres idosas à epidemia do HIV ${ }^{9}$.

Ainda sobre o modo de transmissão, a proporção de ignorados foi de $26,1 \%$. Este alto percentual é uma limitação no conhecimento da real situação da transmissão do HIV.

No presente estudo, evidencia-se predomínio de homens idosos $(78,5 \%)$ com Aids. Contudo, pode-se observar uma oscilação da Aids entre as mulheres, cuja razão alcançou proporção de 12:1. A diminuição da magnitude da razão entre os sexos tem confirmado a manutenção da tendência da feminização da epidemia no país também entre os idosos. Como mostram os dados, a Aids aumenta paulatinamente entre todas as idades no sexo feminino, e as mulheres compõem a maior porcentagem de casos de Aids à medida que aumenta a idade ${ }^{20}$.

Nesta pesquisa, a maior proporção de casos verificou-se no grupo de indivíduos com idades entre 60 e 69 anos (77,5\%). Vale ressaltar que, entre os idosos, esta faixa de idade concentra maior número de pessoas. A maioria dos danos à saúde mostra variação de incidência em função da idade. Entretanto, para aquisição do HIV, há formas de se prevenir. Nesta faixa etária a maior porcentagem de contaminação pelo vírus ocorreu por via sexual e, em sua maioria, entre os heterossexuais. A situação a 
que o indivíduo se expõe é plenamente prevenível, como recomenda a prática de sexo seguro, utilizando-se devidamente o preservativo masculino e feminino nas relações sexuais. Há, porém, referência de que a maioria dos idosos realiza o ato sexual sem a proteção do preservativo, talvez até mesmo por não acreditar que possam contrair Aids e ser contaminados por esta doença ${ }^{21}$.

Associado ao descrédito do idoso de contrair o HIV, segundo observado, dá-se pouca importância à sexualidade do idoso e à Aids nesta faixa etária, pois os velhos são excluídos da sociedade, da comunidade científica e das políticas públicas ${ }^{9}$.

A forma de transmissão do HIV no sexo masculino, quando da ocorrência dos primeiros casos neste estudo (1989 a 1993), é observada na categoria homo-bissexual. No Brasil, a partir de 1998, evidencia-se o aumento e a permanência dos casos entre os sexos na subcategoria heterossexual, sendo esta a que mostrou o maior aumento e a que mais tem contribuído para o processo de feminização, ao influenciar de forma decisiva a expansão da epidemia entre as mulheres ${ }^{22,23}$.

\section{Pauperização}

De acordo com o demonstrado por pesquisa sobre conhecimento relacionado à transmissão do HIV, práticas sexuais e comportamento de vulnerabilidade quanto à infecção pelo HIV, quanto menor o grau de instrução, menor o percentual de acerto sobre conhecimento correto referente às formas de transmissão do HIV ${ }^{24}$.

Conforme revelam determinados estudos, o número de casos aumentou nos estratos de menor escolaridade, remetendo à condição de pior cobertura dos sistemas de vigilância e de assistência médica entre os economicamente menos favorecidos, sob a hipótese de que a escolaridade é uma variável importante de estratificação social ${ }^{25}$.

As populações já tradicionalmente marginalizadas estão cada vez mais se infectando pelo HIV. Como agravante, acrescenta-se a dificuldade de acesso aos serviços de saúde e à informação. Tudo isso somado à necessidade de estarem voltados para a busca de sobrevivência, dificultando as ações destinadas à prevenção por meio da prática do sexo seguro e diagnóstico precoce da doença, questões essas que demonstram as desigualdades socioeconômicas de acesso à informação e recursos de saúde no Brasil ${ }^{19}$.

Neste estudo observa-se que $44,0 \%$ apresentam baixa ou nenhuma escolaridade e que $36,4 \%$ têm grau de estudo ignorado. Isto poderá influir nos resultados encontrados. Essa limitação também se observa em outros estudos realizados no Brasil $^{25}$.

Neste estudo, a análise da distribuição dos casos de Aids, segundo o tamanho populacional dos municípios, mostrou que a capital deteve o maior número de casos, embora o vírus não tenha poupado cidadãos idosos de municípios de pequeno porte.. Em tais localidades, a pobreza e a miséria são constantes, e geram sérias dificuldades de acesso desses indivíduos aos diferentes serviços de saúde, ocasionadas pela distância entre as localidades, pela ausência de profissionais e até pela insatisfatória qualidade dos serviços de saúde oferecidos.

\section{Mortes por Aids}

A Aids é uma das principais causas de morte prematura em diferentes países do mundo. No Brasil, cerca de 50\% dos pacientes ainda morrem no período de até seis meses após o diagnóstico da primeira infecção oportunista. A mortalidade é um dos indicadores mais sensíveis para avaliar o efeito de medidas preventivas e terapêuticas. Neste aspecto, observou-se no Brasil que o acesso gratuito e universal aos anti-retrovirais a partir da segunda metade da década de 1990 reduziu a mortalidade por Aids em $50 \%{ }^{26,27}$.

Como assinalada por alguns autores, a introdução de potentes esquemas antiretrovirais na prática clínica e o emprego rotineiro de tratamentos para infecções 
oportunistas têm sido medidas efetivas de queda da letalidade e morbidade associadas à infecção pelo $\mathrm{HIV}^{28}$.

Entretanto, mesmo na vigência de tratamentos altamente eficazes, a morte constitui ocorrência constante. Nesta pesquisa, a razão entre o número de óbitos por Aids entre homens e mulheres caiu de 2,5 em 1998 para 2,0 em 2003, em virtude do crescimento da letalidade no sexo feminino, que foi de $73,9 \%$.

Essa situação também foi observada no país. De acordo com alguns estudos, a mortalidade por Aids em mulheres, no Brasil, vem aumentando. Na faixa etária de 80 anos e mais, a letalidade é de 100,0\%. Nesta condição, como afirmam alguns autores, idosos com Aids adoecem e morrem mais rápido do que os jovens. Isto decorre do diagnóstico tardio da doença e da combinação de outras enfermidades que possivelmente aceleram a progressão da moléstia ${ }^{6,29}$.

\section{Conclusão}

Algumas tendências caracterizam a epidemia da Aids neste estudo. Observouse uma oscilação na proporção de mulheres, aumento da heterossexualização e da incidência no idoso.

A transmissão heterossexual constituiu a principal fonte de contaminação do HIV entre homens e mulheres idosos, apesar da transmissão entre homens com prática de natureza homo-bissexual ser relevante,

Conforme se conclui, os vários fatos identificados por este estudo devem ser aprofundados sobremaneira, incentivando pesquisas comportamentais de vulnerabilidade relacionadas à infecção pelo HIV em idosos. Desse modo, será possível o monitoramento do comportamento sexual de risco, com vistas à intervenção na epidemia, e, conseqüentemente, redução da morbimortalidade por Aids na terceira idade.

\section{Referências}

1. BRASIL, Ministério da Saúde Secretaria de Vigilância em Saúde. Programa Nacional de DST e AIDS.. Bol Epidemiol AIDS 2005; 2(1): 3-5.

2. Barbosa ASM. Adesão ao tratamento anti-retroviral entre idosos vivendo com Aids na grande São Paulo [tese de doutorado]. São Paulo: Faculdade de Saúde Pública; 2006. Disponível em http://bases.bireme.br/cgi-binwxislind.exe/iah/online/ [acessado em 28 de agosto de 2007].

3. Perez BFA,Gasparini SM. A vivência do idoso no processo de envelhecer e o HIV/Aids: uma reconstrução dupla em suas possibilidades e limites. J Bras Aids 2005; 6(3): 106-9. Disponível em http://bases.bireme.br/cgi-binwxislind.exe/iah/online/ [acessado em 28 de agosto de 2007].

4. CEARÁ. Secretaria da Saúde do Estado do Ceará. Coordenadoria de Políticas em Saúde. Núcleo de Epidemiologia. Informe Epidemiológico Aids. FortalezaCE, 16 de março de 2005 .

5. BRASIL,Ministério da Saúde. Cresce o número de portadores de AIDS na terceira idade. Jornal a Tribuna Digital. Santos, SP. 07/03/2004. Edição eletrônica. Disponível em http://www.AIDS.gov.br/imprensa/ noticias. Impressão. Asp NOT Cod = 54691. [acessado em 02 de março de 2005].
6. De Carlo P. Qué necesitan las personas mayores de 50 años en la prevención del HIV? Disponível em http:// www.caps.ucsf.edu/mayores.htm [acessado em $22 \mathrm{de}$ julho de 2005].

7. Ribeiro A. Sexualidade na terceira idade. In: Papaléo Netto M. Gerontologia. São Paulo: Atheneu; 2006. p.157.

8. EL-Sadr W, Getteler J. Unrecognized human immunodeficiency virus infection in the elderly. JAMA 1995; 155(2).

9. Ceccato MGB, Acurcio FA, Bonelo PF, Rocha GM, Guimarães MDC. Compreensão de informações relativas ao treinamento anti-retroviral entre indivíduos infectados pelo HIV. Cad Saúde Pública 2004; 20(5):138897.

10. BRASIL, Ministério da Saúde. Estatuto do idoso. Brasília; 2003.

11. ORGANIZAÇÃO MUNDIAL DE SAÚDE. Manual de Classificação Estatística Internacional de doenças, lesões e causas de óbito. 9a revisão, 1975. São Paulo: Centro Colaborador da OMS para a Classificação de Doenças em Português; 1985. 
12. ORGANIZAÇÃO MUNDIAL DE SAÚDE. Classificação Estatística Internacional de doenças e problemas relacionados à saúde. 10a revisão. São Paulo: Centro Colaborador da OMS para a Classificação de Doenças em Português; 1995.

13. BRASIL, Ministério da Saúde/Cenepi/datasus. Estatística de mortalidade CD-rom. Rio de Janeiro, out/2000. Declaração de óbito, 1979 a 1998, disponível em HTTP:/ / www.datasus.gov.br [acessado em 28 de junho de 2005].

14. BRASIL. Ministério da Saúde. Secretaria Executiva, DATASUS, Informações em Saúde: mortalidade-SIM. Disponível em www.datasus.gov.br/cgi/ tabecgi.exe?ibge/cnv/pop.ce.def [acessado em $28 \mathrm{de}$ junho de 2005]. 1996 a 2000. CD-rom.

15. Kerr-Pontes LRS, Rouquayrol MZ. A Medida de Saúde Coletiva. In: Rouquayrol MZ, Almeida Filho N de. Epidemiologia e Saúde. 6 ed. Rio de Janeiro: MEDSI; 2003. p. 37-79.

16. BRASIL, Ministério da Saúde. Diretrizes e normas regulamentadoras de pesquisa envolvendo seres Humanos. Brasília; 1996.

17. Santos NJS, Tayra A, Silva SR, Buchalla CM, Laurentini R. A Aids no Estado de São Paulo.As mudanças no perfil da epidemia e perspectivas da vigilância epidemiológica. Rev. Bras. Epidemiol 2002; 5(3).

18. Matsushita RY, Santana RS. Uma análise da incidência dos casos de AIDS por faixa etária. Bol Epidemiol AIDS 2001;15(2): 41-7.

19. Castilho EA, Rodrigues-Junior AL. A epidemia da AIDS no Brasil, 1991-2000. Rev Bras Soc Med Trop 2004; 37(4): 312-

20. BRASIL, Ministério da Saúde. Programa Nacional de DST e AIDS. O perfil da AIDS entre as mulheres do Brasil, América Latina e Caribe. Rev Saber Viver 2005; 5(32): 123.
21. Moriguti JC, Machado AA, Fatori A. Síndrome da Imunodeficiência em Idosos. Gerontologia 1966; 4(3).

22. Rachid M, Schechter M. Manual de HIV/Aids. 6.00. Rio de Janeiro: Revinter; 2001.

23. Buchalla CM, Paiva V. Da compreensão da vulnerabilidade social ao enfoque multidisciplinar. Rev Saude Pub 2002; 36(4): 108-16

24. Szwarcwald CL, Barbosa Junior A, Pascon AR, Sousa Junior PR. Pesquisa de conhecimento, atitudes e práticas na população brasileira de 15 a 54 anos, 2004. Bol Epidemiol AIDS 2004; 13(1): 18-24.

25. Varella, R.B.Aspectos da epidemia de Aids em municípios de médio porte do Rio de Janeiro, 2000 - 2004. Rev Bras Epidemiol 2006; 9(4): 447-53.

26. BRASIL, Fundação Nacional de Saúde. Guia de Vigilância epidemiológica. 6. ed. Brasília-DF FUNASA; 2006. p. 83.

27. Marins JRP, Jamalww LF, Chen S, Hudes ES, Barbosa Junior A, et al. Relatório de Pesquisa: sobrevivência atual dos pacientes com Aids no Brasil, evidência dos resultados de um espaço nacional. Bol Epidemiol AIDS. Brasília 2001/2002; 15(1):37-47.

28. Rachid M, Schechter M. Manual de HIV/Aids. 8. ed. Rio de Janeiro: Revinter; 2005. p. 3-7.

29. Fonseca M, Barreira DA. A evolução da mortalidade por AIDS no país segundo sua distribuição geográfica. Bol Epidemiol AIDS 2000; 13(3). Disponível em http:// www.Aids.gov.br/sitebol.artig02a.htm [acessado em 22 de julho de 2005].

Recebido em: 01/09/06 Versão final reapresentada em: 04/09/07 Aprovado em: 10/09/07 